\title{
Towards explaining publishing activity in Facebook
}

\author{
Jerzy Surma \\ Department of Computer Science and Digital Economy \\ Warsaw School of Economics \\ Warsaw, Poland \\ E-mail: jerzy.surma@sgh.waw.pl
}

\author{
Luvai Motiwalla \\ Operations and Information Systems Department \\ University of Massachusetts \\ Lowell, USA \\ E-mail: luvai_motivalla@uml.edu
}

\begin{abstract}
A growing number of users, seeking to influence their friends, are adopting social network sites, like Facebook to increase their social presence. In the relatively few scholarly studies that consider how writing comments on social network sites influences their friends, the focus has been on marketing or promotion. Little is known about the impact of user writing versus reading on social media. In our paper, we address this gap by studying how the writing comments influences the reactive behavior (likes, comments) of their friends. Our research sheds more light on what motivates users on social networks sites to write and publish on their page. Specifically, we study the user-to-user interactions on Facebook. We found that the number of messages the user broadcasts is dependent on the feedback he or she received from friends.
\end{abstract}

\section{INTRODUCTION}

$\mathrm{L}$ ARGE volumes of status update messages are posted by Facebook (FB) users on their pages to inform their friends and followers about their current activity like eating in restaurant, watching movie or others [1]. They are an increasingly popular form of communication that allow users to interact with their followers who can react with a comment or a "like". Status updates enable quick and effortless one-tomany communication [2]. On average, there are 60 million status updates per day FB making it by far most popular social network site (SNS) and a logical place to study user influence behaviors and consequences of the social processes associated with SNS usage [3]. The popularity and novelty of status updates makes it a very interesting topic for a variety of empirical studies [4].

This paper focuses on understanding users' write or posting activity, which is crucial for the social network site's existence. What reactions are influencing users to publish? Our research finding shows that feedback given by friends and followers has a critical influence on the writing activity. Data obtained from social networks sites are undoubtedly an important source of knowledge about user behaviors and constitute an important source of information for developing a new business models. Facebook thanks to Graph API protocol, provides access to information on the behaviors through consistent view of the social graph with a representation of objects in the graph (e.g., people, photos, events, and pages) and the connections between them (e.g., friend relationships, shared content, and photo tags). Certainly, every user must provide permission to allow access to their data. Social network sites reflect how people act in their social environment. An interesting aspect of living in a society is how an individual's behavior changes under the influence of other people. Social network sites seem to be a natural environment for influencing behaviors captured by SNSs like FB, become a good source for empirical research [3]. This reactive behavior is a powerful organizing standard that governs the social structures and weaves interpersonal webs of connection [5]. Several other studies on social network behavior have found presence of influence in groups with similar age or ethnicity [6].

Status updates are short messages that are posted to the personalized welcome page (News Feed) of all FB friends of the user as well as on the user's own profile page. Most social networking sites, like FB, Google+, and MySpace utilize some form of status updates, and in some cases, like on Twitter, they serve as the main function. These posts are restricted in length (e.g., 420 characters on FB) and recipients can comment on them or indicate that they "like" them. Status updates enable effortless and fast one-to-many communication

This research could be utilized for understanding user behaviors that are useful for market analysis. However, user information must be analyzed with compliance of privacy laws to avoid any individual privacy infringements. Adequate care to anonymize data before publishing the results would be helpful.

In the next section, we provide a brief overview on the related works in the scope of the behavioral research on social networks, with the examples from FB-related studies. Additionally, we have reviewed select studies on user behaviors in social media. In the third section, we discuss our methods of empirical data gathering and provide background information on the research group and variables included in our pilot study. The fourth section presented results yielded from our analysis of empirical data. The final section discusses our conclusions and elaborates on future research opportunities from this work.

\section{RELATED WORK}

Companies today have their social media departments gain insights into the behaviors of their customers through social networks sites like FB, Twitter and others for understanding user behavior on social networks. In addition, social networks sites are ideal grounds for observing influential behaviors that encourage more interactions and affect their followers' opinion [7]. In fact, [8] suggest SNS can be used for rating the relevance of information by using SNS conventions such as 
